\title{
Sex Difference in the Association between Serum Homocysteine Level and Non-Alcoholic Fatty Liver Disease
}

\author{
Bo-Youn Won, Kyung-Chae Park*, Soo-Hyun Lee, Sung-Hwan Yun, Moon-Jong Kim, Kye-Seon Park, \\ Young-Sang Kim, Ji-Hee Haam, Hyung-Yuk Kim, Hye-Jung Kim, Ki-Hyun Park \\ Department of Family Medicine, CHA Bundang Medical Center, CHA University, Seongnam, Korea
}

\begin{abstract}
Background: The relationship between serum homocysteine levels and non-alcoholic fatty liver disease is poorly understood. This study aims to investigate the sex-specific relationship between serum homocysteine level and non-alcoholic fatty liver disease in the Korean population.

Methods: This cross-sectional study included 150 men and 132 women who participated in medical examination programs in Korea from January 2014 to December 2014. Patients were screened for fatty liver by abdominal ultrasound and patient blood samples were collected to measure homocysteine levels. Patients that consumed more than 20 grams of alcohol per day were excluded from this study.

Results: The homocysteine level (11.56 vs. $8.05 \mathrm{nmol} / \mathrm{L}$ ) and the proportion of non-alcoholic fatty liver disease $(60.7 \%$ vs. $19.7 \%)$ were significantly higher in men than in women. In men, elevated serum homocysteine levels were associated with a greater prevalence of non-alcoholic fatty liver disease (quartile 1, 43.6\%; quartile 4, 80.6\%; $\mathrm{P}=0.01$ ); however, in females, there was no significant association between serum homocysteine levels and the prevalence of non-alcoholic fatty liver disease. In the logistic regression model adjusted for age and potential confounding parameters, the odds ratio for men was significantly higher in the uppermost quartile (model 3, quartile 4: odds ratio, 6.78; 95\% confidential interval, 1.67 to 27.56); however, serum homocysteine levels in women were not associated with non-alcoholic fatty liver disease in the crude model or in models adjusted for confounders. Conclusion: Serum homocysteine levels were associated with the prevalence of non-alcoholic fatty liver disease in men.
\end{abstract}

Keywords: Non-Alcoholic Fatty Liver Disease; Homocysteine; Sex Characteristics; Men; Fatty Liver 


\section{INTRODUCTION}

Non-alcoholic fatty liver disease (NAFLD) refers to the presence of hepatic steatosis when no other causes for secondary hepatic fat accumulation, such as significant alcohol consumption, use of steatogenic medication, or hereditary disorders, are present. ${ }^{1)}$ The term NAFLD refers of to a wide spectrum of liver diseases, including simple steatosis, nonalcoholic steatohepatitis (NASH), liver cirrhosis, and hepatocellular carcinoma. ${ }^{2)}$ NAFLD is the most common cause of chronic liver disease in the industrialized world, and the prevalence of NAFLD has become a substantial burden to public health. ${ }^{3)}$

Many studies have reported that NAFLD is associated with metabolic disorders caused by insulin resistance, such as obesity, dyslipidemia, type 2 diabetes, and metabolic syndrome. ${ }^{4,5)}$ Moreover, cardiovascular disease risk factors, such as a high Framingham risk score, carotid intima-media thickness, and coronary artery calcification, are considerably elevated in NAFLD patients. ${ }^{6,7)}$

Homocysteine, a sulfur-containing amino acid, is an intermediate product in the normal biosynthesis of the amino acids methionine and cysteine. ${ }^{8,9)}$ Homocysteine is known to be an independent risk factor for atherosclerosis and cardiovascular disease. The pathogenesis of homocysteine in the development of cardiovascular disease is unclear but possible mechanisms are oxidative stress, lipid peroxidation, and decreased antioxidant enzyme activity. ${ }^{9-12)}$ Moreover, several studies have reported differences in circulating homocysteine concentrations between the sexes; however, lower concentrations in females may be characteristic of the premenopausal years only. ${ }^{13)}$

The liver plays a central role in the metabolism of homocysteine; consequently, liver damage may affect the homocysteine level. Indeed, hyperhomocysteinemia has been reported in chronic alcoholism and in alcoholic liver cirrhosis. ${ }^{14,15)}$ However, the previous data reporting the potential association between serum levels of homocysteine and liver damage in adults with NAFLD are conflicting. ${ }^{8,16)}$ Therefore, the aim of this study is to investigate how sex affects the association between serum homocysteine levels and risk of NAFLD in the Korean population.

\section{METHODS}

\section{Study Population}

This cross-sectional study included adults who participated in the medical examination program, for their health check-up, in South Korea from January 2014 to December 2014. From the fatty liver group and the control group, we excluded patients: that consumed more than 20 grams of alcohol per day; with positive serologic markers for hepatitis B and C; with any chronic liver disease, malignancies, diabetes mellitus, thyroid disease, and renal disease; and that used medication that may induce steatosis (corticosteroids, estrogens, methotrexate, tamoxifen, tuberculostatic drugs) or modified plasma level of homocysteine (folate, vitamin B, C, E, antibiotics). After these exclusions, 117 patients diagnosed with NAFLD and 165 healthy patients without evidence of fatty liver, confirmed by an abdominal ultrasound, were included in the study. All patients who took part in the study signed a written informed consent form, and this study was approved by the institutional review board of CHA Bundang Medical Center in Korea (IRB no. BD2013-081).

\section{General Characteristics}

We conducted research on the medical history and the lifestyle habits of the patients using a self-report questionnaire. Smoking history was used to categorize patients into current smoker and non-smoker groups. Exercise history was used to categorize participants into a routine group (moderate to strenuous intensity, three times a week or more frequently) and a non-routine group. Height and weight were measured with the patient in a standing position without shoes. In this study, body mass index (BMI) represents the patient's weight, in kilograms, divided by the square of their height, in meters. Waist circumference was measured midway between the patient's lower rib margin and their iliac crest with the patient in a standing position. Blood pressure (BP) was measured after the patient had rested for 10 minutes in a sitting position using an automatic sphygmomanometer.

\section{Laboratory Test}

Blood samples were collected in the morning, after the patient had fasted overnight for at least 8 hours. The serum samples were stored at $4^{\circ} \mathrm{C}$ and analyzed within 1 day after sampling. The glucose, liver enzyme, and lipid profiles were tested using an automatic analyzer (HITACHI 7600; Hitachi, Tokyo, Japan). The serum homocysteine was measured with a homocysteine enzymatic kit (Roche Diagnostics, Mannheim, Germany) using an enzymatic assay. The intra- and interassay coefficients were $1.3 \%$ and $2.2 \%$, respectively.

\section{Fatty Liver Assessment}

An abdominal ultrasound was used for the detection and diagnosis of fatty liver; the ultrasound was performed using a 3.5-MHz transducer (General Electric Logiq 7; General Electric Medical Systems, Milwaukee, WI, USA). Experienced radiologists carried out the ultrasound examinations. Images were captured in the standard fashion, with a patient in the supine position with their right arm raised above the head. Hepatic steatosis is defined as the presence of diffuse hyperechoic texture, bright liver, increased liver echo texture compared to the kidneys, vascular blurring, and deep attenuation of the ultrasonic beam. ${ }^{17)}$ The ultrasound results were classified into normal findings and NAFLD (mild and moderate to severe fatty liver) findings.

\section{Statistical Analysis}

All analyses were conducted separately for men and women. The variables were expressed as a mean \pm standard deviation, median (interquartile range), or number (proportion). To compare the variables between the sexes, the independent t-test (for continuous variables) and the chi-square test (for categorical variables) were used. The participants were classified into quartiles according to their serum level of 
homocysteine. We calculated the prevalence of NAFLD within the different quartiles for both sexes using the chi-square test. To assess the influence of homocysteine on risk of NAFLD, logistic regression models were conducted separately for men and women. Model 1 is a crude model. Model 2 was adjusted for age, BMI, current smoking status, routine exercise, systolic $\mathrm{BP}$, diastolic $\mathrm{BP}$, and history of hypertension and dyslipidemia. In addition to the adjustment made in model 2, model 3 was also adjusted for aspartate aminotransferase, alanine aminotransferase, triacylglycerol (logarithmically transformed) and high density lipoprotein cholesterol. A P-value of $<0.05$ was considered to be statistically significant. All statistical analyses were performed using PASW SPSS ver. 18.0 (SPSS Inc., Chicago, IL, USA).

\section{RESULTS}

One hundred fifty men and 132 women participated in the study. The baseline characteristics of the participants are presented in Table 1. The mean age was higher in men than in women ( 42.14 years vs. 39.89 years). The proportion of current smokers ( $40 \%$ vs. $3.8 \%$ ) and the proportion of patients who routinely exercised (30\% vs. $17.4 \%$ ) were higher in men than in women. Hypertension (16\% vs. $6.1 \%$ ) and dyslipidemia (13.3\% vs. $6.1 \%$ ) were more prevalent in men than in women, and BMI (25.11 vs. $22.55 \mathrm{~kg} / \mathrm{m}^{2}$ ) and waist circumference (87.77 vs. $77.31 \mathrm{~cm}$ ) were higher in men than in women. All of the metabolic parameters and laboratory test results differed between men and women

Table 1. Clinical and biochemical characteristics of the study population

\begin{tabular}{|c|c|c|c|}
\hline Characteristic & Men $(n=150)$ & Women ( $n=132)$ & P-value* \\
\hline Age (y) & $42.14 \pm 7.30$ & $39.89 \pm 7.12$ & 0.010 \\
\hline Current smoker & $60(40.0)$ & $5(3.8)$ & $<0.001$ \\
\hline Routine exercise $^{\dagger}$ & $45(30.0)$ & $23(17.4)$ & 0.014 \\
\hline Hypertension & $24(16.0)$ & $8(6.1)$ & 0.009 \\
\hline Dyslipidemia & $20(13.3)$ & $8(6.1)$ & 0.042 \\
\hline Body mass index (kg/m²) & $25.11 \pm 3.30$ & $22.55 \pm 3.79$ & $<0.001$ \\
\hline Waist circumference (cm) & $87.77 \pm 9.40$ & $77.31 \pm 8.64$ & $<0.001$ \\
\hline Systolic BP (mm Hg) & $119.34 \pm 12.28$ & $112.48 \pm 14.25$ & $<0.001$ \\
\hline Diastolic BP (mm Hg) & $74.67 \pm 9.73$ & $69.13 \pm 10.22$ & $<0.001$ \\
\hline Aspartate aminotransferase (IU/L) & $24.55 \pm 15.03$ & $18.76 \pm 6.543$ & $<0.001$ \\
\hline Alanine aminotransferase (IU/L) & $31.76 \pm 27.65$ & $16.36 \pm 11.95$ & $<0.001$ \\
\hline$\gamma$-Glutamyl transferase (U/L) & $34.71 \pm 26.55$ & $16.89 \pm 16.65$ & $<0.001$ \\
\hline \multicolumn{4}{|l|}{ Lipid profile (mg/dL) } \\
\hline Total cholesterol & $204.71 \pm 35.60$ & $196.62 \pm 34.88$ & 0.056 \\
\hline Triglyceride & $156.02 \pm 96.86$ & $92.98 \pm 51.70$ & $<0.001$ \\
\hline High density lipoprotein cholesterol & $45.85 \pm 11.14$ & $58.58 \pm 11.37$ & $<0.001$ \\
\hline Low density lipoprotein cholesterol & $123.88 \pm 29.46$ & $114.07 \pm 31.81$ & 0.008 \\
\hline Homocysteine ( $\mu \mathrm{mol} / \mathrm{L})$ & $11.56 \pm 3.90$ & $8.05 \pm 2.17$ & $<0.001$ \\
\hline Non-alcoholic fatty liver disease (\%) & $91(60.7)$ & $26(19.7)$ & $<0.001$ \\
\hline
\end{tabular}

Values are presented as mean \pm standard deviation or as a number (\%).

$\mathrm{BP}$, blood pressure.

${ }^{*}$ Calculated using the independent $t$-test and $\chi^{2}$-test. ${ }^{\dagger}$ Moderate to strenuous intensity $+\geq 3$ times a week
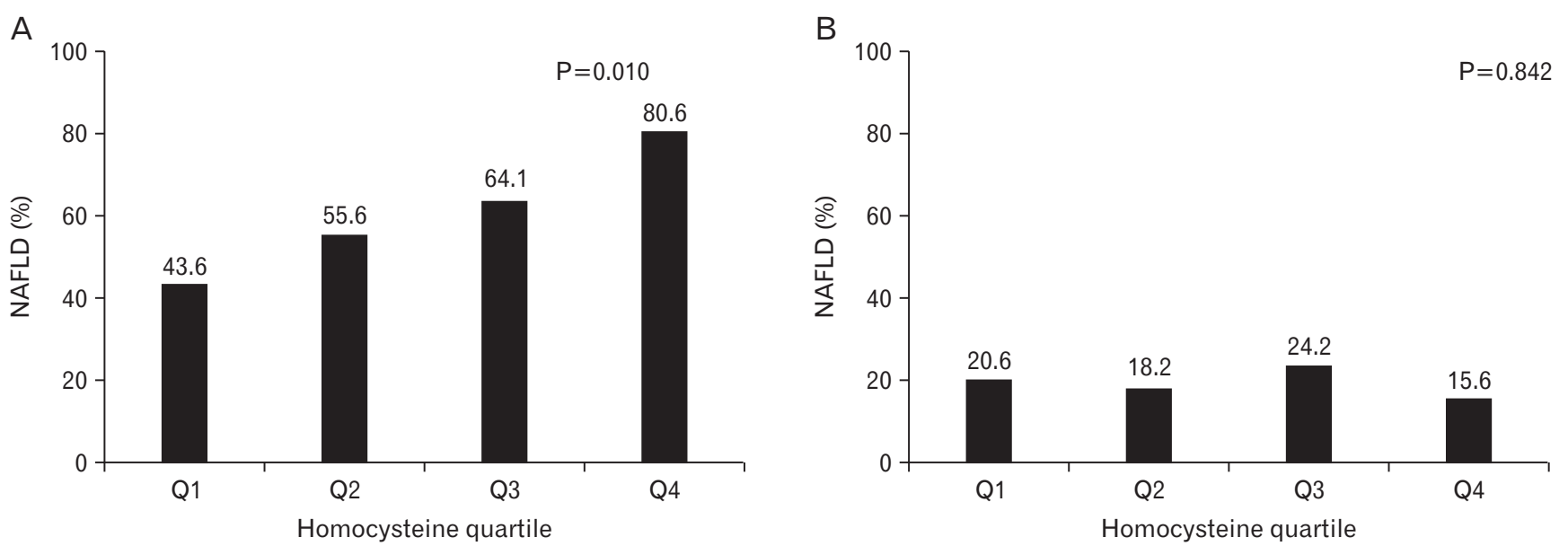

Figure 1. The association between NAFLD and serum homocysteine levels. (A) Men. (B) Women. NAFLD, non-alcoholic fatty liver disease. 
except for total cholesterol. The homocysteine level was significantly higher in men than in women (11.56 vs. $8.05 \mathrm{nmol} / \mathrm{L}$ ), and the proportion of patients with NAFLD was significantly higher for men than for women (60.7\% vs. $19.7 \%)$.

To investigate whether the serum homocysteine level was associated with the prevalence of NAFLD, the homocysteine levels of patients were divided into quartiles. As shown in Figure 1, there was a positive correlation between the prevalence of NAFLD in men and their serum homocysteine level. The prevalence of NAFLD was $43.6 \%$ for men whose serum homocysteine levels were in the first quartile, and the NAFLD prevalence increased to $80.6 \%$ for men whose serum homocysteine levels were in the fourth quartile $(\mathrm{P}=0.01)$ (Figure 1$)$. However, in females, the prevalence of NAFLD was not significantly associated with serum homocysteine levels ( $\mathrm{P}=0.842)$.

The logistic regression model showed the effect of the serum level of homocysteine on the risk of NAFLD (Tables 2, 3). In the crude model (model 1), the odds ratio (OR) for men was significantly higher in the uppermost quartile than in the lowermost quartile of serum homocysteine levels (quartile 4 [Q4]: OR, 5.36; 95\% confidence interval [CI], 1.90 to 15.17). This association between serum homocysteine levels and risk of NAFLD in men was unaffected by adjustments made to the other potential confounding factors (model 2, Q4: OR, 6.35; 95\% CI, 1.80 to 22.52; model 3, Q4: OR, 6.78; 95\% CI, 1.67 to 27.56) (Table 2). In contrast, the serum homocysteine level of women was not associated with risk of NAFLD (model 1, Q4: OR, 0.71; 95\% CI, 0.20 to 2.53) even after adjustment for confounding factors (model 2, Q4: OR, 0.13; 95\% CI,
0.02 to 0.88 ; model 3, Q4: OR, 0.06; $95 \%$ CI, 0.01 to 0.70 ) (Table 3).

\section{DISCUSSION}

We designed our study based on the hypothesis that NAFLD is associated with elevated levels of serum homocysteine. However, in this study, the correlation between homocysteine levels and the prevalence and risk of NAFLD was found to be significant only in men. This may be due to the effect of endogenous sex steroids.

Previously, Leach et al. ${ }^{10)}$ reported similar results to our study. They showed that the serum homocysteine level was significantly higher in patients with biopsy-proven NASH than in patients without biopsyproven NASH, in the control group. They suggested that hyperhomocysteinemia could be attributed to the change in the transsulfuration pathway, a consequence of liver dysfunction. ${ }^{10)}$ By contrast, Polyzos et al. ${ }^{16)}$ reported that a lower serum homocysteine level was observed in NASH patients than in NAFLD patients although homocysteine levels were similar between NAFLD patients and controls. However, the study by Polyzos et al. ${ }^{16)}$ did not examine how the patient's sex affected the association between homocysteine levels and the prevalence of NASH or NAFLD.

Some experimental studies explained that homocysteine might be involved in the pathogenesis of NAFLD. Aissa et al. ${ }^{18)}$ reported that dietary methionine deficiency and methionine supplementation can affect homocysteine levels and higher levels of homocysteine can induce molecular abnormalities in the liver that are associated with the

Table 2. The association between serum homocysteine levels and non-alcoholic fatty liver disease in men

\begin{tabular}{cccc}
\hline \multirow{2}{*}{ Quartile } & Homocysteine $(\mu \mathrm{mol} / \mathrm{L})$ & Model 1 & Odds ratio (95\% confidence interval) \\
\cline { 3 - 3 } Q1 $(n=39)$ & $6.50-9.60$ & 1 & 1 \\
Q2 $(n=36)$ & $9.70-10.90$ & $1.62(0.65-4.03)$ & $1.36(0.40-4.59)$ \\
Q3 $(n=39)$ & $10.94-12.60$ & $2.31(0.93-5.74)$ & $1.88(0.53-6.68)$ \\
Q4 $(n=36)$ & $12.70-46.20$ & $5.36(1.90-15.17)$ & $6.35(1.80-22.52)$ \\
\hline
\end{tabular}

Model 1: no adjustments were made. Model 2: adjusted for age, body mass index, current smoking, routine exercise, systolic BP, diastolic BP, history of hypertension, and dyslipidemia. Model 3: adjusted for aspartate aminotransferase, alanine aminotransferase, triglyceride, and high density lipoprotein cholesterol in addition to adjustments made in model 2.

BP, blood pressure.

*The odds ratios were calculated by logistic regression analyses.

Table 3. The association between serum homocysteine levels and non-alcoholic fatty liver disease in women

\begin{tabular}{cccc}
\hline Quartile & Homocysteine $(\mu \mathrm{mol} / \mathrm{L})$ & Model 1 & Odds ratio (95\% confidence interval)* \\
\cline { 3 - 3 } Q1 $(n=34)$ & $3.80-6.60$ & 1 & Model 2 \\
Q2 $(n=33)$ & $6.70-7.80$ & $0.86(0.26-2.89)$ & 1 \\
Q3 $(n=33)$ & $7.90-9.21$ & $1.23(0.39-3.90)$ & $0.47(0.11-2.08)$ \\
Q4 $(n=32)$ & $9.30-14.80$ & $0.71(0.20-2.53)$ & $0.83(0.21-3.31)$ \\
\hline
\end{tabular}

Model 1: no adjustments were made. Model 2: adjusted for age, body mass index, current smoking, routine exercise, systolic BP, diastolic BP, history of hypertension, and dyslipidemia. Model 3: adjusted for aspartate aminotransferase, alanine aminotransferase, triglyceride, and high density lipoprotein cholesterol in addition to adjustments made in model 2.

$\mathrm{BP}$, blood pressure.

*The odds ratios were calculated by logistic regression analyses. 
development of NAFLD. Matte et al. ${ }^{19)}$ reported that chemically induced hyperhomocysteinemia in rats can induce not only increased oxidative stress and fibrosis but also inflammatory infiltration in the liver.

Some studies have reported differences in the levels of homocysteine between the sexes. Morris et al. ${ }^{13)}$ conducted a study of the general population of women in the US and hypothesized that estrogen status is inversely related to the circulating total homocysteine level, independent of nutritional status and muscle mass. Nakhai Pour et al. ${ }^{20)}$ also reported that there is no significant association between testosterone, estradiol, or dehydroepiandrosterone sulfate levels and plasma homocysteine levels in middle-aged and elderly men. Boers et al. ${ }^{21)}$ suggested that methionine might be catabolized more in premenopausal women than in men by a methionine transamination pathway. Therefore, accumulation of homocysteine, after methionine loading, would be less likely in premenopausal women. ${ }^{21,22)}$ As our female patients were of a child-bearing age, differences in estrogen levels may explain why the total concentrations of homocysteine differed by sex.

There are some limitations of this study. First, the diagnosis of NAFLD was based on ultrasound findings; however, the ultrasound was performed by an expert radiologist. Second, this study was a crosssectional study, and we could not establish a causative relationship between homocysteine and NAFLD. Third, our study was based on a health examination program and some information was collected using self-reported questionnaires, consequently, we had limited accesses to the participants' menopause data. Third, as folate and vitamin B12 levels were not measured, we could not rule out the influence of vitamins on the level of homocysteine. Therefore, additional adjustment for folate and vitamin B12 levels would improve the statistical models.

In conclusion, this is the first study to investigate the relationship between serum homocysteine levels and risk of NAFLD for men and women separately. We found that homocysteine was closely associated with the prevalence of NAFLD in men but not in women.

\section{CONFLICT OF INTEREST}

No potential conflict of interest relevant to this article was reported.

\section{REFERENCES}

1. Chalasani N, Younossi Z, Lavine JE, Diehl AM, Brunt EM, Cusi K, et al. The diagnosis and management of non-alcoholic fatty liver disease: practice Guideline by the American Association for the Study of Liver Diseases, American College of Gastroenterology, and the American Gastroenterological Association. Hepatology 2012;55:2005-23.

2. Matherly SC, Puri P. Mechanisms of simple hepatic steatosis: not so simple after all. Clin Liver Dis 2012;16:505-24.

3. Marino L, Jornayvaz FR. Endocrine causes of nonalcoholic fatty liver disease. World J Gastroenterol 2015;21:11053-76.

4. Kasturiratne A, Weerasinghe S, Dassanayake AS, Rajindrajith S, de Sil- va AP, Kato N, et al. Influence of non-alcoholic fatty liver disease on the development of diabetes mellitus. J Gastroenterol Hepatol 2013;28: 142-7.

5. Boyraz M, Hatipoglu N, Sari E, Akcay A, Taskan N, Ulucan K, et al. Nonalcoholic fatty liver disease in obese children and the relationship between metabolic syndrome criteria. Obes Res Clin Pract 2014;8:e35663.

6. Zhang QQ, Lu LG. Nonalcoholic fatty liver disease: dyslipidemia, risk for cardiovascular complications, and treatment strategy. J Clin Transl Hepatol 2015;3:78-84.

7. Speliotes EK, Massaro JM, Hoffmann U, Vasan RS, Meigs JB, Sahani DV, et al. Fatty liver is associated with dyslipidemia and dysglycemia independent of visceral fat: the Framingham Heart Study. Hepatology 2010;51:1979-87.

8. Gulsen M, Yesilova Z, Bagci S, Uygun A, Ozcan A, Ercin CN, et al. Elevated plasma homocysteine concentrations as a predictor of steatohepatitis in patients with non-alcoholic fatty liver disease. J Gastroenterol Hepatol 2005;20:1448-55.

9. Ganguly P, Alam SF. Role of homocysteine in the development of cardiovascular disease. Nutr J 2015;14:6.

10. Leach NV, Dronca E, Vesa SC, Sampelean DP, Craciun EC, Lupsor M, et al. Serum homocysteine levels, oxidative stress and cardiovascular risk in non-alcoholic steatohepatitis. Eur J Intern Med 2014;25:762-7.

11. Faeh D, Chiolero A, Paccaud F. Homocysteine as a risk factor for cardiovascular disease: should we (still) worry about? Swiss Med Wkly 2006;136:745-56.

12. Zhou J, Austin RC. Contributions of hyperhomocysteinemia to atherosclerosis: causal relationship and potential mechanisms. Biofactors 2009;35:120-9.

13. Morris MS, Jacques PF, Selhub J, Rosenberg IH. Total homocysteine and estrogen status indicators in the Third National Health and Nutrition Examination Survey. Am J Epidemiol 2000;152:140-8.

14. Finkelstein JD. The metabolism of homocysteine: pathways and regulation. Eur J Pediatr 1998;157 Suppl 2:S40-4.

15. Cravo ML, Gloria LM, Selhub J, Nadeau MR, Camilo ME, Resende MP, et al. Hyperhomocysteinemia in chronic alcoholism: correlation with folate, vitamin B-12, and vitamin B-6 status. Am J Clin Nutr 1996;63: 220-4.

16. Polyzos SA, Kountouras J, Patsiaoura K, Katsiki E, Zafeiriadou E, Deretzi G, et al. Serum homocysteine levels in patients with nonalcoholic fatty liver disease. Ann Hepatol 2012;11:68-76.

17. Sanyal AJ; American Gastroenterological Association. AGA technical review on nonalcoholic fatty liver disease. Gastroenterology 2002;123: 1705-25.

18. Aissa AF, Tryndyak V, de Conti A, Melnyk S, Gomes TD, Bianchi ML, et al. Effect of methionine-deficient and methionine-supplemented diets on the hepatic one-carbon and lipid metabolism in mice. Mol Nutr Food Res 2014;58:1502-12.

19. Matte C, Stefanello FM, Mackedanz V, Pederzolli CD, Lamers ML, Dutra-Filho CS, et al. Homocysteine induces oxidative stress, inflammatory infiltration, fibrosis and reduces glycogen/glycoprotein content in liver of rats. Int J Dev Neurosci 2009;27:337-44.

20. Nakhai Pour HR, Grobbee DE, Muller M, Emmelot-Vonk M, van der Schouw YT. Serum sex hormone and plasma homocysteine levels in middle-aged and elderly men. Eur J Endocrinol 2006;155:887-93. 
21. Boers GH, Smals AG, Trijbels FJ, Leermakers AI, Kloppenborg PW. Unique efficiency of methionine metabolism in premenopausal women may protect against vascular disease in the reproductive years. J Clin Invest 1983;72:1971-6.
22. Kim MH, Kim E, Passen EL, Meyer J, Kang SS. Cortisol and estradiol: nongenetic factors for hyperhomocyst(e)inemia. Metabolism 1997;46: 247-9. 